Erratum

\title{
Erratum to: Volume of a doubly truncated hyperbolic tetrahedron
}

Alexander Kolpakov and Jun Murakami

Erratum to: Aequat. Math. 85 (2013), 449-463

DOI 10.1007/s00010-012-0153-y

In the original publication of the article, formulas (2), (14) and (17) contain some errors.

The first named author bears the responsibility for these errors. He would like to thank Prof. Atakan T. Yakut (Nigde University), who drew the authors' attention to this fact.

The correct version of formulas (2), (14) and (17) are given below,

1. The matrix $G^{\star}$ from formula (2) should read as

$$
G^{\star}=\left(\begin{array}{cccc}
-1 & -\cos \mu & i \sinh \ell_{5} & i \sinh \ell_{3} \\
-\cos \mu & -1 & i \sinh \ell_{6} & i \sinh \ell_{2} \\
-i \sinh \ell_{5} & -i \sinh \ell_{6} & -1 & -\cosh \ell_{1} \\
-i \sinh \ell_{3} & -i \sinh \ell_{2} & -\cosh \ell_{1} & -1
\end{array}\right)
$$

2. In formula (14), the function $\mathscr{E}$ should read as

$$
\mathscr{E}=\phi\left(z_{-}\right) \psi\left(z_{+}\right)\left(c_{34}+\delta \frac{a_{1}-1 / a_{1}}{2}\right)-\phi\left(z_{+}\right) \psi\left(z_{-}\right)\left(c_{34}-\delta \frac{a_{1}-1 / a_{1}}{2}\right)
$$

3. Consequently, formula (17) should have the form

$$
\Re \frac{\partial \mathscr{V}}{\partial \theta_{1}}=-\frac{1}{4} \log \left|\frac{c_{34}-\delta \frac{a_{1}-a_{1}^{-1}}{2}}{c_{34}+\delta \frac{a_{1}-a_{1}^{-1}}{2}}\right| .
$$

The online version of the original article can be found under doi:10.1007/s00010-012-0153-y. 
All the errors listed above do not affect the result of the original publication of the article.

\author{
Alexander Kolpakov \\ Department of Mathematics \\ University of Fribourg \\ chemin du Musée 23 \\ 1700 Fribourg, Switzerland \\ e-mail: kolpakov.alexander@gmail.com \\ Jun Murakami \\ Department of Mathematics \\ Faculty of Science and Engineering \\ Waseda University \\ 3-4-1 Okubo Shinjuku-ku \\ Tokyo 169-8555, Japan \\ e-mail: murakami@waseda.jp
}

